\title{
Chemical Interference of Pathogen-associated Molecular Pattern-triggered Immune Responses in Arabidopsis Reveals a Potential Role for Fatty-acid Synthase Type II Complex-derived Lipid Signals ${ }^{*[5}$
}

Received for publication, September 12, 2006, and in revised form, December 12, 2006 Published, JBC Papers in Press, December 13, 2006, DOI 10.1074/jbc.M608792200

Mario Serrano $^{\ddagger}$, Silke Robatzek ${ }^{\ddagger}$, Martha Torres ${ }^{\ddagger}$, Erich Kombrink ${ }^{\ddagger}$, Imre E. Somssich ${ }^{\ddagger}$, Mike Robinson $^{\S}$, and Paul Schulze-Lefert ${ }^{\ddagger}$

From the ${ }^{\ddagger}$ Department of Plant-Microbe Interactions, Max Planck Institute for Plant Breeding Research, Carl-von-Linné Weg 10, 50829 Cologne, Germany and ${ }^{\S}$ Syngenta Jealott's Hill International Research Centre, Bracknell,

Berkshire RG42 6EY, United Kingdom

We describe an experimental setup using submerged cultures of Arabidopsis seedlings in 96-well microtiter plates that permits chemical intervention of rapid elicitor-mediated immune responses. Screening of a chemical library comprising 120 small molecules with known biological activities revealed four compounds reducing cellulysin- or flg22-activated gene expression of the early pathogen-associated molecular patterns (PAMP)responsive $A T L 2$ gene. One chemical, oxytriazine, was found to induce $A T L 2$ gene expression in the absence of PAMP. By monitoring additional flg22-triggered immediate early plant responses, we present evidence that two compounds, triclosan and fluazinam, interfere with the accumulation of reactive oxygen species and internalization of the activated plasma membrane resident FLS2 immune receptor. Using triclosan structure types and enzyme activity inhibition assays, Arabidopsis MOD1 enoyl-acyl carrier protein reductase, a subunit of the fatty-acid synthase type II (FAS II) complex, was identified as a likely cellular target of triclosan. Inhibition of all tested elicitor-triggered early immune responses by triclosan indicates a potential role for signaling lipids in flg22-triggered immunity. Chemical profiling of eca mutants, each showing deregulated ATL2 gene expression, with the identified compounds revealed mutantspecific response patterns and allowed us to deduce tentative action sites of $E C A$ genes relative to the compound targets.

In plants and animals, the first active defense response against microbial invasion is initiated upon the recognition of pathogen-associated molecular patterns (PAMPs). ${ }^{2}$ These patterns, also referred to as elicitors, include components of fungal

* This work was supported by a Max Planck post-doctoral fellowship (to M. S.) and in part by Fonds der Chemischen Industrie, Germany. The costs of publication of this article were defrayed in part by the payment of page charges. This article must therefore be hereby marked "advertisement" in accordance with 18 U.S.C. Section 1734 solely to indicate this fact.

5 The on-line version of this article (available at http://www.jbc.org) contains supplemental Figs. 1-4 and Table 1.

'To whom correspondence should be addressed. Tel.: 49-221-5062-350; Fax: 49-221-5062-353; E-mail: schlef@mpiz-koeln.mpg.de.

${ }^{2}$ The abbreviations used are: PAMP, pathogen-associated molecular pattern; LPS, lipopolysaccharide; PRR, pattern recognition receptor; FAS II, fattyacid synthase type II; ACP, acyl carrier protein; ACC, acetyl-CoA carboxylase; DHFR, dihydrofolate reductase; GUS, $\beta$-glucuronidase. cell walls such as chitin ( $N$-acetyl-chito-oligosaccharide oligomers), lipopolysaccharides (LPS) from the outer membrane of Gram-negative bacteria, flagellin, and EF-Tu $(1,2)$. The signaling pathways and molecular mechanisms underlying PAMP-triggered innate immunity are not completely elucidated. Genetic and biochemical evidence points to an intimate link between PAMP-triggered immunity and the production of reactive oxygen species, ligand-induced endocytosis of pattern recognition receptors (PRRs), protein phosphorylation, mitogen-activated protein kinase signaling, and transcriptional activation of the early response gene $(1,3-7)$.

ATL2 is a member of the Arabidopsis ATL gene family and has been characterized as an early and transiently activated PAMP-responsive gene $(8,9)$. Several members of the ATL gene family, each encoding RING-H2 finger domain-containing proteins, are induced by components of fungal cell walls (10) as well as by bacterium-derived flagellin $(4,11)$. Additionally, a tobacco cell culture expressing the tomato resistance gene $C f 9$, a member of the $A T L$ tobacco family, designated ACRE-132, was rapidly induced within 30 min upon addition of the Cladosporium fulvum-derived fungal effector Avr9 in the presence of cycloheximide (12). This suggests that ACRE-132 gene activation occurs independent of de novo protein biosynthesis, a typical feature of early PAMP-responsive genes. A mutational approach aimed at the identification of genetic components directing Arabidopsis ATL2 elicitor-responsive gene induction revealed several mutants showing constitutive expression of ATL2 (eca, expression constitutiva de ATL2) but failed to recover nonresponsive mutant plants (13).

Traditional forward genetic approaches have been widely used to identify and assign genes from various model organisms to specific biological processes. Major limitations of such mutational approaches are lethal mutations because of essential gene functions, pleiotropic effects caused by multifunctionality of a gene product, and redundant gene functions. Small molecules with inducing or inhibitory activities have the potential to complement mutational approaches for the dissection of biological processes because chemical intervention can be performed in a conditional, dose-dependent, and reversible manner (14). A model organism for plant biology, Arabidopsis thaliana, provides the potential to probe small molecule libraries for com- 


\section{Molecules Modulating PAMP-triggered Innate Immune Responses}

pounds acting on cellular targets that are inaccessible to conventional mutational analysis (15). Few studies have systematically explored the potential of small molecules to interfere with plant-specific processes (16-18).

Here we describe an experimental system that permits chemical manipulation of early PAMP-triggered immune responses in Arabidopsis seedlings. By taking advantage of several transgenic lines each carrying a fusion between an early PAMP-responsive promoter and the $\beta$-glucuronidase reporter gene, we screened a chemical library comprising 120 small molecules with known biological activities. Submerged cultures of Arabidopsis seedlings enabled uniform contact with and controlled exposure of the plants to the elicitors cellulysin or flg22 as well as to the tested compounds. By monitoring $\beta$-glucuronidase reporter activity histochemically and/or in quantitative fluorimetric assays, we identified compounds that either inhibit or induce early PAMP-responsive genes. The candidate compounds were subsequently tested for their ability to interfere with elicitor-induced reactive oxygen species production and PRR endocytosis. The likely cellular target of one of the inhibitory compounds, enoyl-ACP reductase, was validated by the use of structure variants and enoyl-ACP reductase activity assays. Our results indicate a possible role for signaling lipids in PAMP-triggered immune responses and provide the basis for future screening of natural compound libraries.

\section{EXPERIMENTAL PROCEDURES}

Plant Material-In this study we used Arabidopsis transgenic lines carrying fusions of the following $5^{\prime}$-regulatory gene sequences to the $\beta$-glucuronidase (GUS) reporter gene: $A T L 2$ (8), WRKY22, WRKY29, and 4CL1 (described below). ATL2p::GUS is expressed in a C-24 background, WRKY22p::GUS and WRKY29p::GUS in Col-0, and 4CL1p::GUS in a Landsberg erecta background. The transgenic Ws-0/FLS2-GFP line used for monitoring FLS2 endocytosis is described by Robatzek et al. (6).

GUS Reporter Constructs-1.8 and $1.9 \mathrm{~kb}$ of the $5^{\prime}$ upstream region (relative to the ATG start codon) of the AtWRKY22 (At4g01250) and AtWRKY29 (At4g23550) locus, respectively, were PCR-amplified from Arabidopsis Col-0 genomic DNA using Gateway suitable primers, 5' -TTACCTACATGTGCTTTGCTGTTGC-3' and 5' -ATCGTCGGCCATTGAATTTGGTTAC-3' (AtWRKY22) and 5' -TACTCACAGAAGTCAATAAGCGCG-3' and 5' -CGACTATTGCTTCTAGGTCTCCTTC-3' (AtWRKY29), and cloned into the binary pJAWOHL11-GW-GUS vector resulting in a translational fusion with the uidA gene. $1 \mathrm{~kb}$ of the $5^{\prime}$ upstream region of the At4CL1 (At1g51680) locus (19) was PCR-amplified from genomic Arabidopsis Col-0 genomic DNA using the following primers: B11F, $5^{\prime}$-ATTAACTGCAGACACTTTTAGCCCATAACTTTC-3' , and B11R, 5' -CTTGTGGCGCCATGGTAAATAGTAAATATTGTG- ${ }^{\prime}$. Primer B11F contained a $5^{\prime}$ PstI restriction sequence, and primer B11R, spanning the ATG codon of the $4 C L 1$ gene, contained one mismatch to the sequence of the At4CL1 gene to generate an NcoI restriction site. For fusion to the uidA gene, the PCR product was digested with PstI and NcoI and ligated with PstI/NcoI-digested vector pBT10-TATA-GUS (20), from which the promoter::GUS cassette was excised with the restriction enzymes ClaI and SacI and inserted into the binary vector pGPTV-Kan (21). Transformations were performed by the dip floral method (22), and transformants were selected by kanamycin resistance.

Growth Conditions and Compound Screening Procedure-The small molecule library was provided by SYNGENTA Corp. Surface-sterilized seeds were stratified for 4 days at $4{ }^{\circ} \mathrm{C}$. Seedlings were grown for 7 days in 96-well microtiter plates (NUNC) containing $\sim 2-4$ seeds per well in Murashige and Skoog (MS) liquid medium and $0.25 \%$ sucrose. The microtiter plates were incubated at $24{ }^{\circ} \mathrm{C}$ with continuous shaking at $100 \mathrm{rpm}$ in a growth chamber (Percival AR-75L) under 16/8 light/dark conditions. After 7 days, the medium was removed by a multichannel pipette (Matrix) and replaced by MS medium containing 10 ppm of each compound dissolved in $\mathrm{Me}_{2} \mathrm{SO}$ and incubated for $120 \mathrm{~min}$. At the end of the preincubation time, seedlings were treated for 120 min using MS medium or MS medium containing $100 \mathrm{mg} / \mathrm{ml}$ cellulysin (Calbiochem) as described previously (8). FLS2-dependent immune responses were induced using MS medium containing $1 \mu \mathrm{M}$ flg22 peptide.

GUS Expression Assay-Histochemical and fluorometric assays were performed as described (23). Samples were mounted on microscope slides using 50\% glycerol and inspected with a Leica MZFLIII stereomicroscope. Protein concentrations were determined by Bradford assays using a commercial kit (Bio-Rad).

Oxidative Burst Assay-Production of reactive oxygen species was assayed by $\mathrm{H}_{2} \mathrm{O}_{2}$-dependent luminescence of luminol (24). Briefly, Arabidopsis leaf pieces $(\sim 1 \mathrm{~mm})$ were floated overnight in water, incubated in a solution containing $1 \%$ $\mathrm{Me}_{2} \mathrm{SO}$ and $10 \mathrm{ppm}$ compound for $30 \mathrm{~min}$ in the presence of luminol and peroxidase, and elicited with $1 \mu \mathrm{m}$ flg22. Luminescence emission was measured in a luminometer (top count NXT; PerkinElmer Life Sciences).

flg22-induced Endocytosis Assay-We analyzed flg22-induced endocytosis as described previously (6). Briefly, detached leaves of Ws-0/FLS2-GFP transgenic seedlings grown under sterile conditions were preincubated in a solution containing $1 \% \mathrm{Me}_{2} \mathrm{SO}$ for $30 \mathrm{~min}$. After adding the tested compounds at 10 ppm for $30 \mathrm{~min}$, endocytosis was triggered by supplying the solution with $10 \mu \mathrm{M}$ flg22 for $30 \mathrm{~min}$. Microscopy was performed using the confocal laser microscope Leica TCS SP2 with a $\times 63$ oil objective and $\times 4$ zoom.

Enoyl-ACP Reductase Activity Assay-Enoyl-ACP reductase activity was measured as described previously (25). Total protein was extracted from ground seedlings using $10 \mathrm{~mm}$ sodium phosphate (pH 9.0) and $1 \mathrm{~mm}$ dithioerythritol. Protein concentration was determined by the Bradford assay using a commercial kit (Bio-Rad). $50 \mu \mathrm{g}$ of protein was dissolved in the reaction buffer (10 mm sodium phosphate ( $\mathrm{pH}$ 6.2) and $140 \mu \mathrm{M}$ NADH). Enoyl-ACP reductase activity was monitored by the decrease of absorbance at $340 \mathrm{~nm}$ because of oxidation of NADH at room temperature $\left(22^{\circ} \mathrm{C}\right) .120 \mu \mathrm{M}$ crotonoyl-CoA (Sigma) was used as substrate, whereas the endogenous NADH oxidation was measured in the absence of crotonoyl-CoA. The reaction was initiated after addition of substrate and was continuously monitored for $15 \mathrm{~min}$. 


\section{RESULTS}

Structurally Diverse Small Molecules Interfere with Early PAMP-responsive Gene Expression-We used a chemical library comprising 120 small molecules with known biological activities (supplemental Table 1) to identify candidate compounds that modified elicitor-responsive gene expression. We established a compound screening procedure using 7-day-old Arabidopsis seedlings grown in submerged culture in 96-well microtiter plates to monitor transcriptional activation of early PAMP-responsive genes (see "Experimental Procedures"). Such genes typically encode transcripts, which transiently increase in abundance within minutes upon PAMP treatment (11). We used a transgenic line carrying a fusion of the early PAMP-response gene ATL2 promoter to the $\beta$-glucuronidase reporter gene (ATL2p::GUS; see Ref. 13). Although constitutive $A T L 2$ gene expression is mainly confined to leaf primordia and the leaf vasculature, treatment with the fungus-derived proteinaceous cellulysin elicitor (8) resulted in visible GUS expression within $2 \mathrm{~h}$ disseminating throughout the seedlings, which corresponds to an $\sim 4$-fold GUS activity increase in total seedling extracts (Fig. 1A). Only 1 of the 120 compounds tested, oxytriazine, a dihydrofolate reductase inhibitor, was capable of simulating cellulysin-mediated reporter gene expression (Fig. $1, A$ and $B$ ), which might be because of an agonist-like oxytriazine activity, spurious $A T L 2$ reporter gene activation via a PAMP-independent signaling pathway, or inhibition of a repressor blocking constitutive $A T L 2$ induction in the authentic cellulysin-triggered signaling pathway. Simultaneous treatment of the seedlings with oxytriazine and cellulysin did not increase GUS reporter gene expression above the level seen with either compound alone. In time course experiments, a more rapid reporter gene activation was detected upon treatment with the cellulysin elicitor than with oxytriazine (induction detectable within 30 and $120 \mathrm{~min}$, respectively; see supplemental Fig. 1). Together this is consistent with the idea that oxytriazine either inhibits a repressor or induces a component of the authentic PAMP-triggered signaling pathway (Fig. 1, $A$ and $B$ ).

We identified four compounds that inhibited cellulysin-triggered GUS reporter activity either strongly ( $\sim 70 \%$; triclosan) or more moderately ( $\sim 50 \%$; fluazinam, cantharidin, and fenpiclonil) (Fig. 1, $A$ and $B$ ). Exposure of the seedlings to varying concentrations of these small molecules revealed a dose-dependent mode of action between 0.1 and 20 ppm (supplemental Fig. 2). Because histochemically detectable GUS reporter activities in leaf primordia were essentially indistinguishable in the absence or presence of each of these four compounds (Fig. 1A), their effect is likely to reflect inhibition of GUS reporter gene activation rather than constitutive GUS reporter gene transcription or GUS enzyme activity modification. Consistent with this, reverse transcription-PCR experiments using seedling-derived RNA revealed a significant reduction of cellulysin-stimulated ATL2 endogene expression upon treatment with each of the compounds (supplemental Fig. 3). Time course experiments showed different inhibition tendency suggesting a more complex mode of action (supplemental Fig. 1). Although a 2-h preincubation period of the seedlings with triclosan and the subsequent addition of cellulysin resulted in maximal and
A
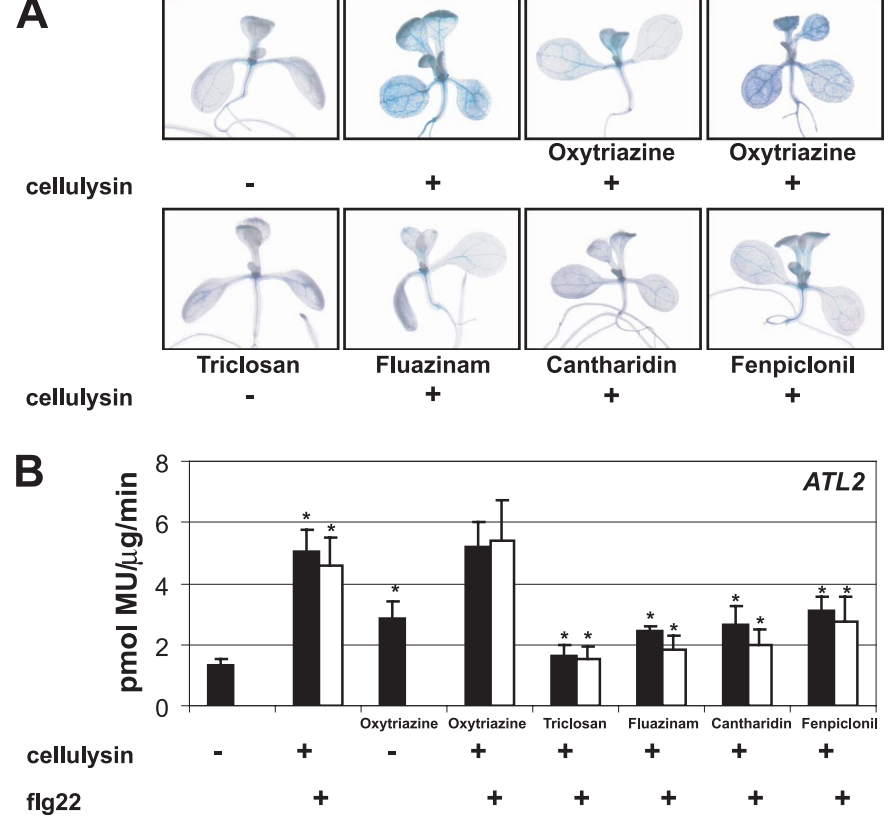

C

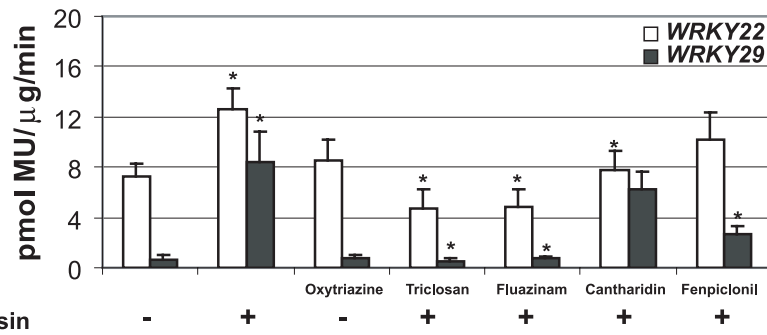

D

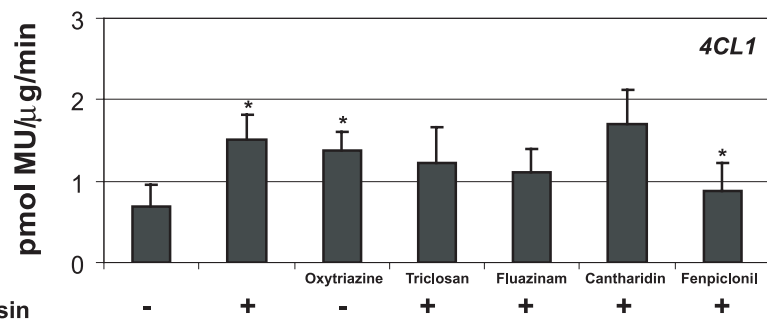

FIGURE 1. Identification of small molecules interfering with early PAMPresponsive gene expression. $A$, histochemical localization of GUS reporter gene activity. Seven-day-old ATL2p::GUS seedlings were preincubated for 120 min with $10 \mathrm{ppm}$ of each indicated compound and induced for $120 \mathrm{~min}$ with MS media or MS media containing $100 \mathrm{mg} / \mathrm{ml}$ cellulysin; representative samples of three independent experiments are shown. B, fluorometric GUS activity assays of ATL2p::GUS seedlings treated with cellulysin (black bars) or $1 \mu \mathrm{M}$ flg22 (white bars). C, WRKY22p::GUS (white bars) and WRKY29p::GUS (black bars). $D, 4 C L 1 p:: G U S$. Treatments in $B-D$ were identical to those described in $A$. Data points shown are the mean of eight independent experiments \pm S.D. Asterisks indicate a significant difference $(p<0.01)$ of GUS activity changes mediated by the indicated compounds relative to the nontreated control (in case of oxytriazine) or cellulysin/flg22-induced samples.

sustained inhibition of reporter gene activity within $30 \mathrm{~min}$, both cantharidin and fenpiclonil permitted additional slight increases of reporter gene activity even 120 min after elicitor addition. Moreover, in the presence of triclosan, fluazinam, or fenpiclonil, basal GUS reporter activity remained unaffected within the 2-h preincubation period, and cantharidin significantly stimulated reporter activity in the absence of cellulysin (120-min time point; supplemental Fig. 1). This might indicate the existence of multiple cellular cantharadin targets exerting 
DMSO

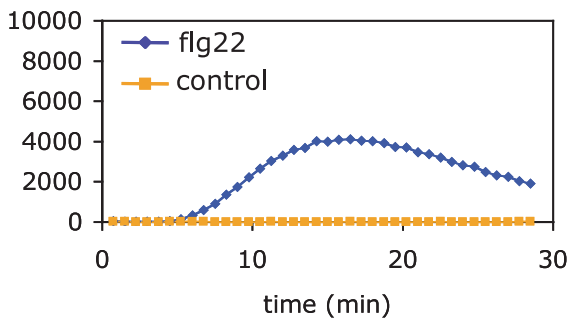

Triclosan

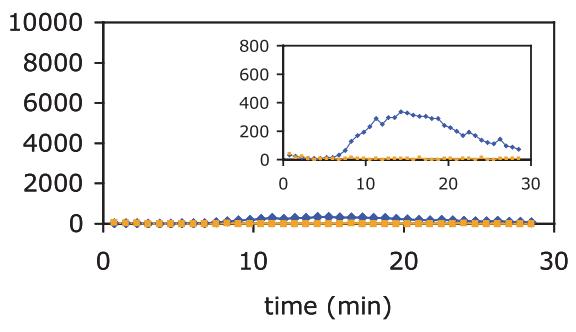

Cantharidin

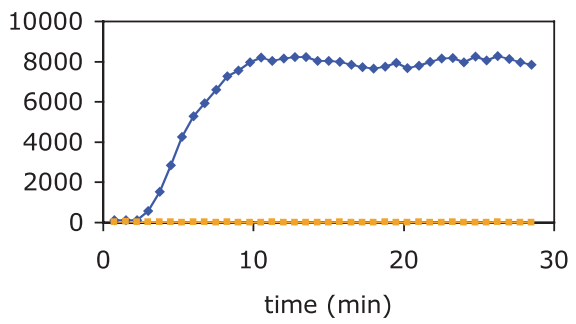

Oxytriazine

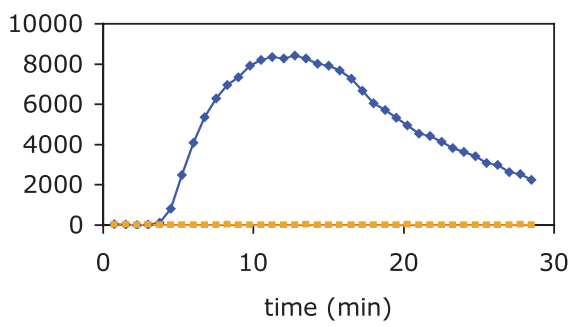

Fluazinam

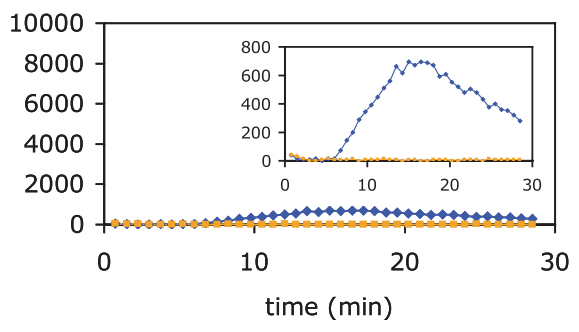

Fenpiclonil

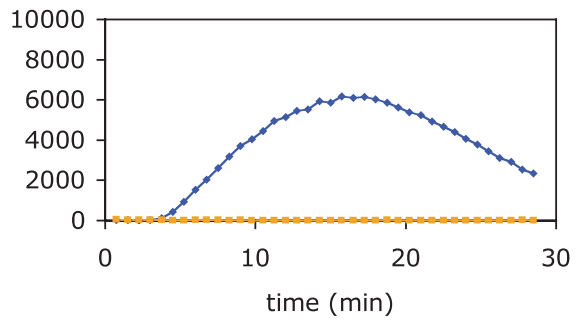

FIGURE 2. Chemical intervention of flg22-dependent production of reactive oxygen species. $A$. thaliana ecotype Landsberg erecta leaf pieces were incubated in a solution containing $1 \% \mathrm{Me}_{2} \mathrm{SO}$ (DMSO) and $10 \mathrm{ppm}$ of the indicated small molecules for $30 \mathrm{~min}$, supplied with luminol and peroxidase, and elicited with $1 \mu \mathrm{m}$ flg22. Luminescence emission is given as relative light units and continuously monitored over the indicated time course. Each graph shows a representative measurement of three independent experiments each with six replicates.

opposite effects on constitutive and PAMP-induced ATL2 gene expression, respectively. Taken together, of the four candidate inhibitors identified, only triclosan, fluazinam, and fenpiclonil appeared to specifically interfere with cellulysin-triggered ATL2 gene expression.

When the ATL2p::GUS-expressing seedlings were treated with flg22, a 22-mer peptide derived from the bacterial motor protein (26), we observed a similar 4-fold reporter gene induction in comparison with cellulysin treatment (Fig. 1B). Each of the five compounds affecting cellulysin-stimulated ATL2 reporter gene activity also interfered with flg22-induced reporter gene activation in an essentially identical manner (Fig. $1 B$ ), suggesting that these small molecules alter the activity of common PAMP-triggered signaling events shared between the two sequence-unrelated elicitors.

To determine whether the identified five compounds affect the induction of early PAMP-responsive genes other than ATL2, we tested additional GUS reporter lines. The triclosan and fluazinam inhibitors of cellulysin-stimulated ATL2p::GUS reporter activity affected in a comparable manner reporter gene activation in WRKY22p::GUS and WRKY29p::GUS transgenic lines ( $\sim 60$ and $80 \%$, respectively; Fig. $1 C$ ). However, fenpiclonil and cantharidin did not significantly reduce reporter gene pendent oxidative burst (26) in leaf tissue in the presence and absence of the identified small molecules to gain further insights in their potential mode of action on immediate early elicitor responses (luminescence assay; see "Experimental Procedures"). None of the five compounds, including the inducer oxytriazine, provoked an oxidative burst in the absence of flg22 (Fig. 2). Interestingly, the flg22-triggered transient oxidative burst was enhanced in the presence of oxytriazine. Thus, oxytriazine can itself induce early PAMP-responsive gene expression but cannot trigger the production of reactive oxygen species. In addition, although its effect leads to a further increase of the elicitor-induced oxidative burst, this is not the case for early PAMP-responsive gene expression (compare Figs. $1 B$ and 2).

Triclosan, the most potent inhibitor of early PAMP-responsive gene expression, also inhibited the flg22-dependent oxidative burst most strongly (about 90\% inhibition; Fig. 2). Tested triclosan structure types exerted similar effects (see below and supplemental Fig. 4). Likewise, fluazinam strongly inhibited the production of flg22-stimulated reactive oxygen species, but in the presence of fenpiclonil, the oxidative burst was hardly affected. Interestingly, cantharidin, which appears to exert opposite effects on constitutive and PAMP-induced ATL2 gene expression, mediated a sustained rather than transient produc- 


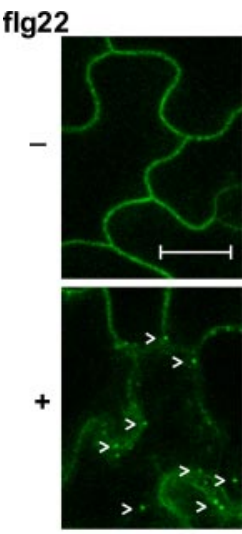

control

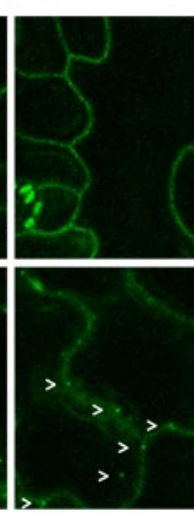

Oxytriazine
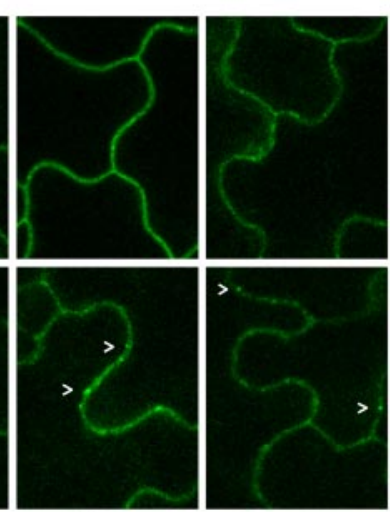

Fluazinam

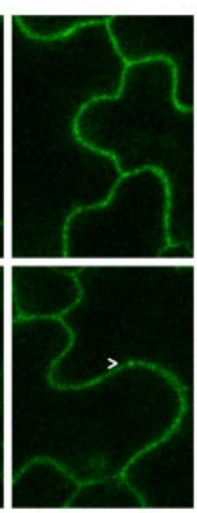

Cantharidin

FIGURE 3. Chemical inhibition of flg22-induced FLS2-GFP endocytosis. Leaves of sterile-grown Ws-0/FLS2 GFP transgenic seedlings were preincubated in a solution containing $1 \% \mathrm{Me}_{2} \mathrm{SO}(\mathrm{DMSO})$ for 30 min. The indicated compounds were added at 10 ppm for $30 \mathrm{~min}$. FLS2-GFP endocytosis was triggered by supplying 10 $\mu \mathrm{M}$ flg22 for $30 \mathrm{~min}$. Micrographs show representative confocal images; bar, $20 \mu \mathrm{m}$. Arrowheads indicate vesicles.

tion of reactive oxygen species (Fig. 2). These complex cantharidin-mediated changes on both the flg22-dependent oxidative burst and PAMP-responsive gene expression are consistent with the presumed multiple cellular targets for this known phosphatase inhibitor and indicate that phosphatase function may be necessary for the transient behavior of the flg22-triggered oxidative burst.

Inhibition of flg22-mediated FLS2 Endocytosis-Recently, flg22-induced endocytosis of its cognate receptor kinase FLS2 has been reported (6). We addressed potential effects of the identified small molecules on PAMP receptors by monitoring FLS2 expression, subcellular localization, and flg22-triggered FLS2 activation. The latter can be assayed indirectly by flg22dependent receptor internalization via endocytosis. Arabidopsis Ws-0 leaves, stably expressing the FLS2-GFP fusion protein (6), were elicited with flg22 after preincubation with the compounds, and FLS2-GFP endocytosis was observed by confocal microscopy. In the absence of flg22, none of the chemicals, including the inducer oxytriazine, led to changes in FLS2-GFP expression levels or subcellular localization within a pretreatment period of $30 \mathrm{~min}$ (Fig. 3). Thus, neither induction by oxytriazine nor chemical suppression of flg22-responsive gene expression by triclosan, fluazinam, cantharidin, and fenpiclonil is because of mis-localization, mis-expression, or degradation of the FLS2 receptor.

Interestingly, the inducer oxytriazine was not capable of triggering FLS2 endocytosis. This suggests that oxytriazine bypasses PAMP receptor activation to elicit PAMP-responsive gene expression (see "Discussion"). Consistent with this, flg22mediated endocytosis of FLS2-GFP was retained in the presence of oxytriazine (Fig. 3). In contrast, flg22-mediated FLS2 endocytosis was clearly affected in the presence of the inhibitors triclosan, fluazinam, and cantharidin (Fig. 3, lower panel). FLS2 endocytosis was retained in the presence of fenpiclonil (Fig. 3, lower panel) but was significantly affected upon longer preincubation times $(1.5 \mathrm{~h}$; data not shown). Although the appearance of FLS2-GFP-containing "buds" at the cell membrane was still detectable in the presence of all the compounds, subsequent steps of endocytosis, such as vesicle "pinching off" and

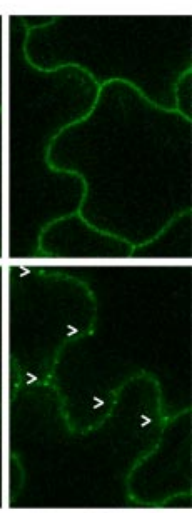

Fenpiclonil mobile FLS2-GFP vesicle trafficking, were severely reduced (Fig. 3).

Chemical Interference of FAS II Leads to Inhibition of Early PAMPresponsive Gene Expression-Fatty acid biosynthesis is an essential metabolic pathway and involves mainly two enzyme complexes, acetyl-CoA carboxylase (ACCase), which catalyzes the carboxylation of acetyl-CoA to malonyl-CoA, and the fatty-acid synthase complex (FAS), catalyzing de novo biosynthesis of fatty acids using malonyl-CoA as the elongator by sequential addition of 2-carbon units to the growing acyl chain (30). In plants, the fatty-acid synthase complex is termed FAS II and consists of eight dissociable polypeptides, each with separate enzymatic activities (Fig. 4A). In Escherichia coli, triclosan targets the fabI gene product, a rate-limiting subunit of the FAS II complex, the enoyl-acyl carrier protein reductase catalyzing the final reduction of the chain elongation cycle (31). Plant enoyl-ACP reductase can functionally replace its counterpart within the bacterial complex as shown by genetic complementation (32). The Arabidopsis enoyl-ACP reductase is encoded by $M O D 1$ and a point mutation in mod1-1 was shown to lead to decreased enzymatic activity, growth, and developmental aberrations as well as spontaneous cell death (33).

We reasoned that if MOD1 is the target of triclosan in Arabidopsis, then other known inhibitors of FAS II or the ACCase complex should exert similar effects on PAMP-triggered ATL2p::GUS reporter gene expression. However, we failed to inhibit ACCase activity in planta using the well characterized inhibitors dichlorofopmethyl, fluazifop acid, or tralkoxidim (Fig. 4B). This might be explained by the selectivity of these inhibitors against the homomeric ACCase form present in monocotyledonous plants, whereas Arabidopsis contains a heteromeric ACCase complex (34). Unlike this, when we targeted FAS II complex components other than enoyl-ACP reductase by treatment of the seedlings with hexachlorophene and cerulenin, we observed a significant inhibition of cellulysin-mediated ATL2p::GUS reporter activity (60 and 40\%, respectively; Fig. $4 B$ ). These results point to a contribution of multiple FAS II complex components, including MOD1 to early PAMP-responsive gene expression.

MOD1 Is a Cellular Target of Triclosan and Triclosan Structure Types-Triclosan structure types inhibiting E. coli enoylACP reductase have been described (35). Each of three tested structural triclosan analogs strongly inhibited cellulysin-mediated ATL2p::GUS reporter activity, although with different effectiveness, demonstrating that the effect of triclosan on reporter gene expression can be modulated by side group modifications of the structural core. The fact that triclosan and the three tested structure types each target the bacterial enoyl-ACP reductase and exert a similar effect on PAMP-triggered reporter gene expression suggests the existence of a common 


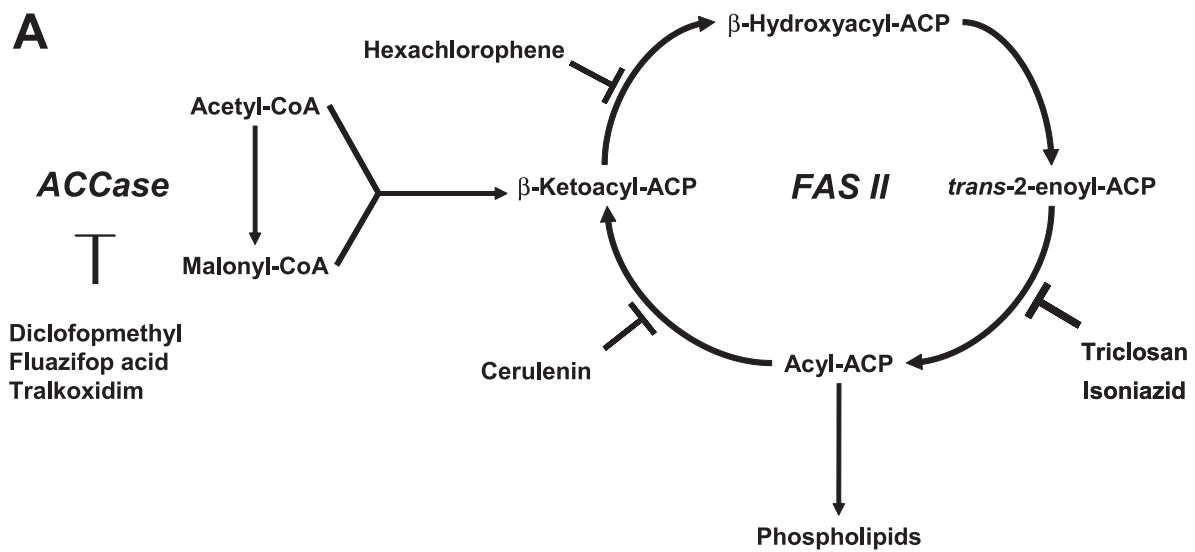

B

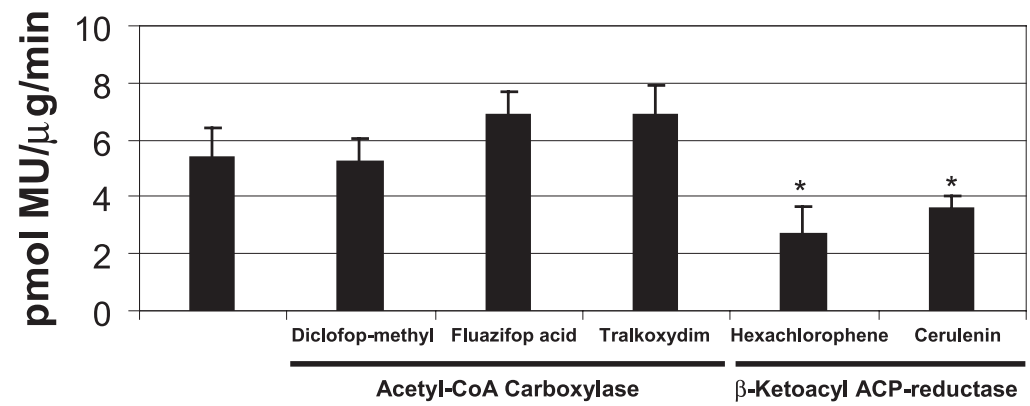

FIGURE 4. Chemical interference of the fatty acid biosynthesis pathway. $A$, fatty acid biosynthesis pathway scheme. Biosynthesis steps blocked by ACCase or FAS II inhibitors are shown. B, fluorometric GUS activity assays. Seven-day-old ATL2p::GUS seedlings were preincubated for $120 \mathrm{~min}$ with $10 \mathrm{ppm}$ of each indicated ACCase or FAS II inhibitor, and immune responses were induced for $120 \mathrm{~min}$ with $100 \mathrm{mg} / \mathrm{ml}$ cellulysin. Data points are the mean of five independent experiments \pm S.D. Asterisks indicate a significant difference $(p<0.01)$ of GUS activity changes mediated by the indicated compounds relative to the cellulysin-induced sample.

cellular target in planta, thereby identifying Arabidopsis MOD1 enoyl-ACP reductase as the likely target (Fig. $5 A$; see also below). Isoniazid is also known to inhibit bacterial enoylACP reductase by a mode of action that is distinct from triclosan (structurally unrelated compound; see Ref. 36). Unexpectedly, isoniazid failed to inhibit cellulysin-triggered ATL2p::GUS reporter activity in Arabidopsis (Fig. 5A). To test whether the differential ability of triclosan and isoniazid to inhibit reporter gene expression correlates with expected changes in MOD1 enzyme activities, we determined in doseresponse experiments in vitro MOD1 activity in plant extracts (compare Fig. 5, $B$ and $C$ ). Although triclosan strongly inhibited MOD1 at both 10 and $20 \mathrm{ppm}$, isoniazid failed to inhibit this enzyme activity even at $20 \mathrm{ppm}$ (Fig. $5 \mathrm{C}$ ). This provides direct evidence that MOD1 is a cellular target of triclosan and reveals an unexpected insensitivity of the plant enzyme to isoniazid.

Chemical Profiling of eca Mutants Suggests Tentative Action Sites of ECA Genes-Previously we described several mutants showing heightened constitutive ATL2 expression (eca (13)). We utilized these mutants to obtain further insights how the identified small molecules might interfere with PAMP-responsive ATL2 gene expression. In the hydroponic growth system used for testing chemical interference of ATL2 promoterdriven reporter gene expression, eca2 and eca5 mutants showed a 2 -fold and eca4 plants more than 4-fold elevated GUS reporter activity relative to wild type (Fig. 6). In each mutant, GUS reporter activity could be further increased by treatment with the cellulysin elicitor. Treatment of the mutants with the inducer oxytriazine revealed a significant increase of the reporter gene induction only in eca2 plants (Fig. 6).

Next we investigated the ability of the inhibitors triclosan, fluazinam, cantharidin, and fenpiclonil to interfere with constitutive or cellulysin-induced GUS reporter gene expression in eca mutants. Upon treatment with the compounds, constitutive reporter gene expression was not affected in the mutant plants (data not shown). Remarkably, cellulysin-induced reporter gene activity in eca4 mutants was completely unaffected by each of these inhibitors (Fig. 6), suggesting that ECA4 acts either downstream of their cellular targets or that loss of ECA4 leads to the activation of a spurious $A T L 2$ induction pathway that is insensitive to the tested compounds. In contrast, cellulysinstimulated reporter gene activity in eca2 plants retains its sensitivity to all but one inhibitor (cantharidin). Because the identified compounds specifically interfere with PAMPinducible but not constitutive ATL2 gene expression (see Fig. 1), this could indicate that ECA2 acts upstream of the triclosan, fluazinam, and fenpiclonil targets. Unlike this, in eca5 plants a significant inhibition of elicitorstimulated GUS reporter activity was detectable in the presence of triclosan and fenpiclonil (Fig. 6), suggesting that cellulysinstimulated signaling is modified in eca5 plants such that is has become insensitive to fluazinam and cantharidin targets. This hints at a potential action of ECA5 downstream of fluazinam and cantharidin targets.

\section{DISCUSSION}

Here we described an experimental setup using 96-well microtiter plates and submerged culture conditions that permits chemical intervention of elicitor-mediated immune responses in a whole organism context. Unlike soil drench or agar media, the use of liquid media allows direct and uniform contact of all seedling organs to the chemicals, thereby minimizing the time interval needed for the compounds to reach their cellular targets (e.g. organ-specific uptake and intercellular as well as intracellular transport rates). This maximizes the probability to intervene with cue-dependent plant processes in a synchronous manner. By this means, we were able to shorten the pretreatment time interval with the chemicals to 120 and 30 min (GUS expression and oxidative bust/flg22-induced endocytosis assays, respectively) before adding the tested elicitors to trigger immune responses (cellulysin or flg22). Because we focused on early elicitor-triggered plant responses in this study, the total exposure time of the seedlings to the chemicals did not 


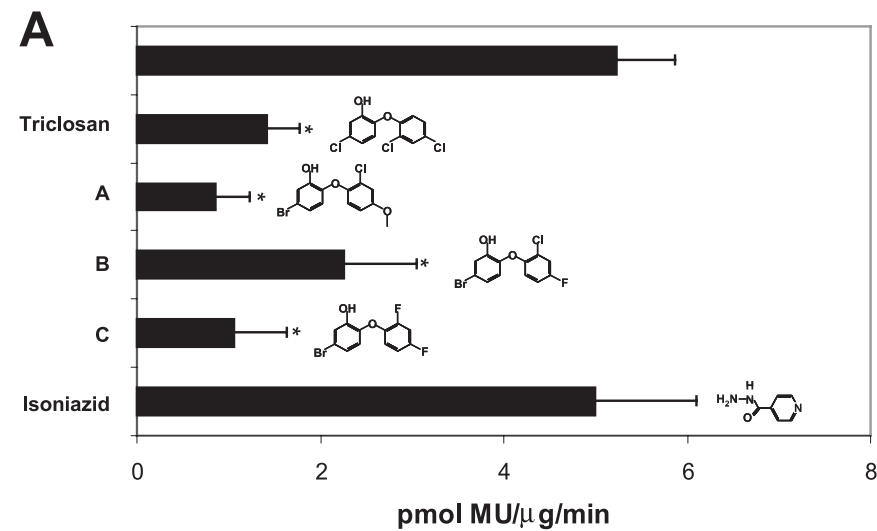

B

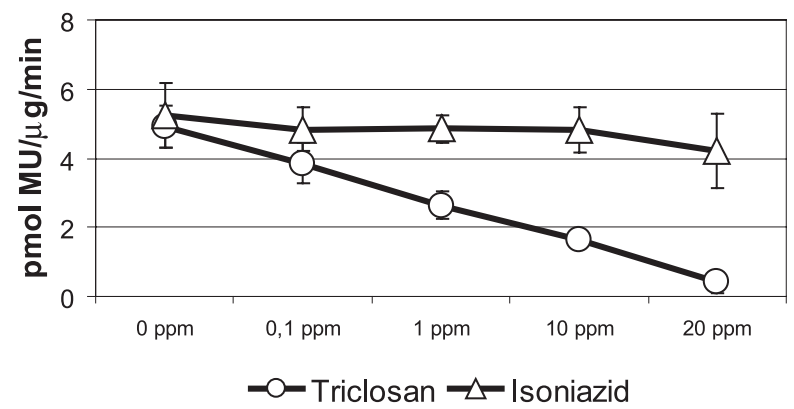

C

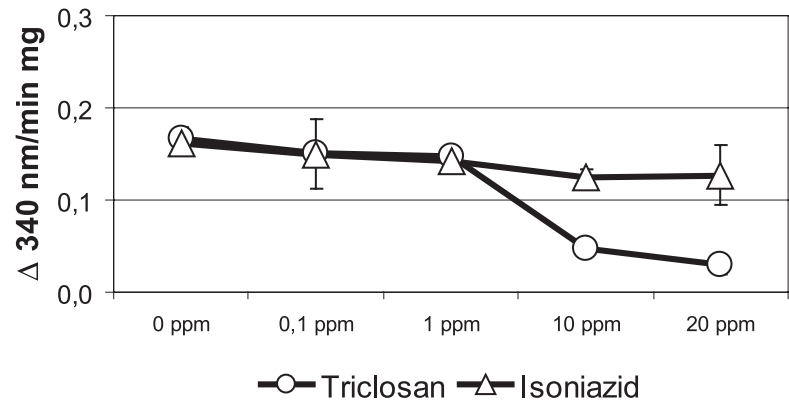

FIGURE 5. Chemical inhibition of enoyl-ACP reductase. $A$, fluorometric GUS activity assays of 7-day-old ATL2p::GUS seedlings preincubated for $120 \mathrm{~min}$ with $10 \mathrm{ppm}$ of each indicated enoyl-ACP reductase inhibitor and induced for 120 with $100 \mathrm{mg} / \mathrm{ml}$ cellulysin. The chemical structures of each tested inhibitor are shown. Data points are the mean of eight independent experiments \pm S.D. Asterisks indicate a significant difference $(p<0.01)$ of GUS activity changes mediated by the indicated compounds relative to the cellulysininduced sample. $B$, fluorometric GUS activity assays; $C$, enoyl-ACP reductase activity assays. Seven-day-old ATL2p::GUS seedlings were treated for $120 \mathrm{~min}$ with the indicated concentrations of triclosan $(O)$ or isoniazid $(\Delta)$. Immune responses were induced for $120 \mathrm{~min}$ with $100 \mathrm{mg} / \mathrm{ml}$ cellulysin. Data points are the mean of three independent experiments \pm S.D.

exceed $4 \mathrm{~h}$ (e.g. for the GUS reporter gene assays). We consider this important because short exposure times can help to minimize secondary or indirect effects of a compound on a biological process. For example, triclosan has herbicidal activity after prolonged exposure (several days) of plants, which is likely because of a physiological collapse resulting from sustained fatty acid biosynthesis inhibition. We hypothesize that the observed rapid (within 30 min; supplemental Fig. 1) triclosandependent inhibition of PAMP-mediated immune responses (early PAMP-responsive gene expression, oxidative burst, inhibition of FLS2 internalization) indicates a signaling rather than structural role of lipids in plant immunity (see below).

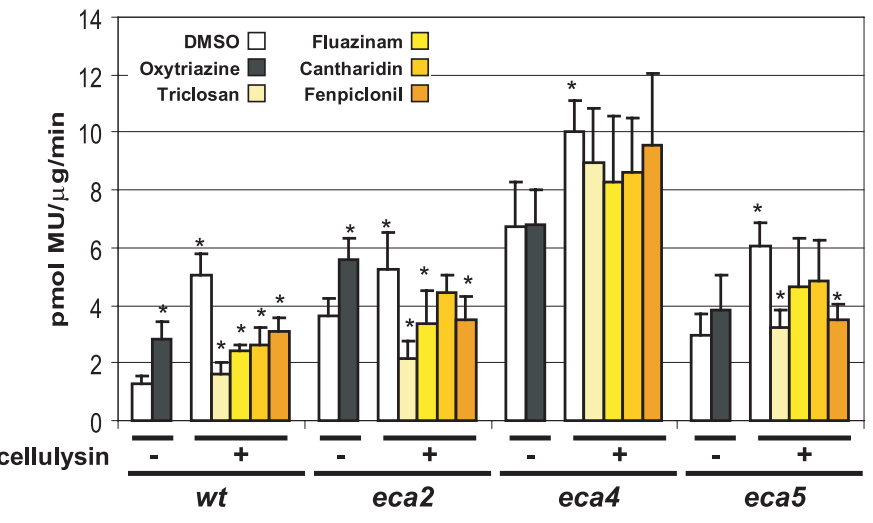

FIGURE 6. Chemical profiling of eca mutants. Fluorometric GUS activity assays of seedlings were preincubated for $120 \mathrm{~min}$ with $10 \mathrm{ppm}$ of each indicated small molecule. Immune responses were induced for 120 min with 100 $\mathrm{mg} / \mathrm{ml}$ cellulysin. Data points are the mean of seven independent experiments \pm S.D. Asterisks indicate a significant difference $(p<0.01)$ of GUS activity changes mediated by the indicated compounds relative to the nontreated control (in case of oxytriazine) or to cellulysin-induced samples in wild type $(w t)$ and eca mutant backgrounds.

The sole compound identified, which is able to induce normally PAMP-responsive gene induction of $A T L 2$ and $4 C L 1$, was oxytriazine, a dihydrofolate reductase (DHFR) inhibitor. DHFR is a key enzyme for the biosynthesis of purines and several amino acids (37). Interestingly, DHFR inhibition was recently shown to stimulate in Drosophila melanogaster gene expression of cell cycle, signal transduction, and immune response genes, indicating the participation of DHFR-derived products in different pathways/responses (38). Because simultaneous treatment of Arabidopsis seedlings with oxytriazine and cellulysin did not increase ATL2p::GUS reporter expression above the level seen with either compound alone (Fig. $1 B$ ) and because oxytriazine on its own neither induced an oxidative burst nor receptor internalization (Figs. 2 and 3), its inducing activity might result from inhibition of a repressor acting in a common PAMP-triggered signaling pathway downstream of receptor activation/endocytosis. We note that the inducing activity of oxytriazine is distinct from other recently identified natural compounds that hyper-induce early PAMP-responsive gene expression only in the presence of the elicitor. ${ }^{3}$ Although oxytriazine alone was unable to provoke an oxidative burst, the chemical clearly enhanced the flg22-dependent production of reactive oxygen species (Fig. 2). One possibility to rationalize this finding is that inappropriate (i.e. premature) inhibition of the inferred PAMP signaling repressor generates a positive feedback signal, thereby amplifying the flg22-dependent oxidative burst. Alternatively, potentiation of the flg22-dependent oxidative burst in the presence of oxytriazine could be due to inhibition of a second cellular target that dampens the production of reactive oxygen species as soon as the oxidative machinery is activated.

In previous mutational studies of cellulysin-triggered ATL2p::GUS activation, we identified several eca mutants showing constitutive ATL2 expression (13). However, despite the screening of large numbers of mutagenized ATL2p::GUS seedlings (50,000 M2 families), we were unable to recover

\footnotetext{
${ }^{3}$ M. Serrano and P. Schulze-Lefert, unpublished data.
} 


\section{Molecules Modulating PAMP-triggered Innate Immune Responses}

mutants that specifically failed to induce ATL2p::GUS expression upon cellulysin treatment. This may be due to genetic redundancy and/or lethality of presumed receptor(s) and downstream signaling factors. In this context, we consider the identification of four chemical inhibitors of early PAMP-responsive gene induction noteworthy because it indicates the potential of conditional chemical inhibition as a complementary approach to mutational analysis. Except for cantharidin that also affects cellulysin-independent constitutive GUS reporter gene expression, the inhibitors triclosan, fluazinam, and fenpiclonil appear to specifically inhibit flg22 or cellulysintriggered $A T L 2 p:: G U S$ induction (Fig. $1 B$ and supplemental Fig. 1). Moreover, whereas fenpiclonil reduced PAMP-triggered gene activation of reporter gene constructs ATL2p::GUS, WRKY29p::GUS, and 4CL1p::GUS, triclosan and fluazinam were unable to inhibit cellulysin-stimulated 4CL1p::GUS induction (compare Fig. 1, B-D). Likewise, oxytriazine only activated ATL2 and $4 C L 1$ gene expression. This reveals an unexpected diversity of factors directing gene expression at different early PAMP-responsive promoters. One possibility is that the observed differential compound sensitivity of the tested reporter gene constructs reflects differences in the composition of cis-acting elements in the respective $5^{\prime}$ regulatory sequences.

The identification of cellular targets of a small molecule is normally a challenging and long term undertaking. By monitoring multiple elicitor-triggered early plant responses in the presence or absence of the compounds (reporter gene induction, oxidative burst, and PRR endocytosis), we were able to delimit their potential site(s) of action. Of the three seemingly specific inhibitors, two, triclosan and fluazinam, impaired both FLS2 endocytosis (Fig. 3) and the flg22-induced oxidative burst (Fig. 2). Thus, the identified chemicals may decrease elicitor-responsive gene induction by interfering with endocytic processes of PAMP receptors at the cell periphery.

Perception of flg22 by the plasma membrane-localized FLS2 receptor kinase almost immediately leads to the generation of reactive oxygen species, protein phosphorylation, mitogen-activated protein kinase signaling, and transcriptional gene activation $(3,4,39)$ and triggers FLS2 internalization by endocytosis (6). FLS2 endocytosis was impaired by the phosphatase inhibitor type 2A cantharidin (Fig. 3). As a potential target in this process, the kinase-associated phosphatase has been shown to interact with FLS2 (40) and is also known to play a role in receptor endocytosis (41). Because substitution of a conserved potential phosphorylation site in the FLS2 receptor kinase (Ws-0/FLS2 ${ }^{\mathrm{T} 876 \mathrm{~V}}$ ) and the general kinase inhibitor (K252a) impairs both receptor endocytosis and all tested FLS2dependent immune responses (6), the observed cantharidin sensitivity of FLS2 endocytosis points to the involvement of dynamic phosphorylation and dephosphorylation events in this process. In addition, we found in the presence of cantharidin a sustained rather than transient accumulation of reactive oxygen species upon flg22 treatment (Fig. 2). It is possible that this deregulated PAMP response is a consequence of the cantharidin-mediated inhibition of FLS2 endocytosis. For example, inhibition of endocytosis of the human TLR4 PRR recognizing bacterial LPS increased LPS signaling (7). Accordingly, plasma membrane localization of activated FLS2 would be sufficient to trigger an oxidative burst, whereas elicitor-responsive gene expression might require receptor internalization.

Triclosan was identified in this work as a potent inhibitor of all tested elicitor-triggered immune responses (elicitor-activated gene expression, FLS2 receptor endocytosis, and flg22triggered oxidative burst). The close correlation between triclosan dose-dependent inhibition of MOD1 activity in plant extracts and reduction of elicitor-triggered ATL2p::GUS induction in planta (Fig. 5, $B$ and $C$ ) is consistent with the idea that Arabidopsis MOD1 enoyl-ACP reductase is a cellular target. Because three tested triclosan structure types, each known to inhibit E. coli enoyl-ACP reductase, mimic in planta the immune response inhibition seen with triclosan (Fig. $5 \mathrm{~A}$ ), we conclude that MOD1 enoyl-ACP reductase is the relevant cellular target of these compounds. Why are the elicitor-stimulated immune responses sensitive to MOD1 enoyl-ACP reductase inhibition, a subunit of the FAS II complex that is localized in plastids? Since a 30-min preincubation period with triclosan was sufficient to compromise ligand-induced FLS2 receptor internalization from the plasma membrane as well as the flg22dependent oxidative burst (Figs. 2 and 3), we consider it unlikely that MOD1 enoyl-ACP reductase inhibition results from a depletion of structural lipids. Unlike structural lipids, signaling lipids such as phosphatidic acid are present in minute amounts and are subject to rapid turnover (reviewed in Ref. 42). In tomato cell cultures, phosphatidic acid and its phosphorylated derivative diacylglycerol pyrophosphate are known to transiently accumulate within minutes upon treatment with flg22 or other elicitors (43). Likewise, phospholipase C inhibitors block the elicitor-induced oxidative burst in tobacco cells (44). Thus, we speculate that inhibition of enoyl-ACP reductase activity by triclosan affects signaling lipids, which in turn might impair elicitor-triggered immune responses. In this context it is interesting that in humans phospholipase D was recently shown to directly interact with and to stimulate the GTPase domain of dynamin, a protein mediating receptor endocytosis through GTP hydrolysis (45). Constitutive expression of a MOD1 mutant variant in mod1-1 plants was shown to reduce MOD1 wild type activity by $50 \%$, leading to pleiotropic growth and developmental anomalies as well as spontaneous cell death (33). In this context it is not surprising that a $72 \%$ reduction of MOD1 activity in the presence of $10 \mathrm{ppm}$ triclosan (Fig. $5 \mathrm{C}$ ) has major effects on elicitor-triggered immunity. The conditional chemical interference of MOD1 activity described here illustrates the potential in revealing putative links between the activity of a multifunctional enzyme complex and signaling lipids in plant immune responses.

To obtain further information where the identified compounds might interfere with PAMP-responsive gene expression, we have taken advantage of previously identified eca mutants showing constitutive ATL2 expression (13). The recessive inheritance of the mutations in eca plants suggests that constitutive ATL2 gene expression results either from the elimination of repressors acting in the authentic elicitor-triggered gene induction pathway or opens spurious signaling pathways enhancing constitutive ATL2 expression. Nonresponsiveness of eca 4 mutants to each identified inhibitor of PAMP-triggered 
ATL2p::GUS reporter gene expression strongly suggests that $E C A 4$ acts downstream of the respective compound targets (Fig. 6). However, we are unable to discriminate whether ECA4 acts in the authentic elicitor-triggered ATL2 gene activation pathway or prevents other spurious signaling pathways to act on ATL2. Nonresponsiveness of elevated ATL2p::GUS reporter activity in eca4 plants to triclosan, fluazinam, cantharidin, and fenpiclonil inhibition also rules out the possibility that under our experimental conditions the compounds generally downregulate gene expression. Unlike $E C A 4$, we consider $E C A 2$ as a candidate acting in the elicitor-triggered ATL2 gene induction pathway because three of the four identified inhibitors (triclosan, fluazinam, and fenpiclonil) reduced cellulysin-stimulated GUS activity in eca 2 plants. Taken together these experiments illustrate the potential to assign genes of a given biological process in tentative pathways according to their sensitivity/insensitivity to chemical inhibitors in much the same way as intermutant crosses are utilized to reveal gene interactions.

Acknowledgments-We thank Dr. Plinio Guzman (CINVESTAVIrapuato, Mexico) for providing the ATL2p::GUS line and eca mutants, Drs. B. Ülker and A. Zhou for WRKY22p::GUS and WRKY29p::GUS reporter lines, and Petra Köchner for technical support.

\section{REFERENCES}

1. Nürnberger, T., Brunner, F., Kemmerling, B., and Piater, L. (2004) Immunol. Rev. 198, 249-266

2. Zipfel, C., and Felix, G. (2005) Curr. Opin. Plant Biol. 8, 353-360

3. Asai, T., Tena, G., Plotnikova, J., Willmann, M. R., Chiu, W. L., GomezGomez, L., Boller, T., Ausubel, F. M., and Sheen, J. (2002) Nature 415, 977-983

4. Zipfel, C., Robatzek, S., Navarro, L., Oakeley, E. J., Jones, J. D., Felix, G., and Boller, T. (2004) Nature 428, 764-767

5. Gross, A., Kapp, D., Nielsen, T., and Niehaus, K. (2005) New Phytol. 165, 215-226

6. Robatzek, S., Chinchilla, D., and Boller, T. (2006) Genes Dev. 20, 537-542

7. Husebye, H., Halaas, O., Stenmark, H., Tunheim, G., Sandanger, O., Bogen, B., Brech, A., Latz, E., and Espevik, T. (2006) EMBO J. 25, 683-692

8. Salinas-Mondragon, R. E., Garciduenas-Pina, C., and Guzman, P. (1999) Plant Mol. Biol. 40, 579-590

9. Gutierrez, R. A., Ewing, R. M., Cherry, J. M., and Green, P. J. (2002) Proc. Natl. Acad. Sci. U. S. A. 99, 11513-11518

10. Serrano, M., Parra, S., Alcaraz, L. D., and Guzman, P. (2006) J. Mol. Evol. 62, 434- 445

11. Navarro, L., Zipfel, C., Rowland, O., Keller, I., Robatzek, S., Boller, T., and Jones, J. D. (2004) Plant Physiol. 135, 1113-1128

12. Durrant, W. E., Rowland, O., Piedras, P., Hammond-Kosack, K. E., and Jones, J. D. (2000) Plant Cell 12, 963-977
13. Serrano, M., and Guzman, P. (2004) Genetics 167, 919-929

14. Smukste, I., and Stockwell, B. R. (2005) Annu. Rev. Genomics Hum. Genet. 6, 261-286

15. Blackwell, H. E., and Zhao, Y. (2003) Plant Physiol. 133, $448-455$

16. Zhao, Y., Dai, X., Blackwell, H. E., Schreiber, S. L., and Chory, J. (2003) Science 301, 1107-1110

17. Armstrong, J. I., Yuan, S., Dale, J. M., Tanner, V. N., and Theologis, A. (2004) Proc. Natl. Acad. Sci. U. S. A. 101, 14978-14983

18. Surpin, M., Rojas-Pierce, M., Carter, C., Hicks, G. R., Vasquez, J., and Raikhel, N. V. (2005) Proc. Natl. Acad. Sci. U. S. A. 102, 4902-4907

19. Ehlting, J., Büttner, D., Wang, Q., Douglas, C. J., Somssich, I. E., and Kombrink, E. (1999) Plant J. 19, 9-20

20. Sprenger-Haussels, M., and Weisshaar, B. (2000) Plant J. 22, 1-8

21. Becker, D., Kemper, E., Schell, J., and Masterson, R. (2000) Plant Mol. Biol. 20, 1195-1197

22. Clough, S. J., and Bent, A. F. (1998) Plant J. 16, 735-743

23. Jefferson, R. A. (1987) Plant Mol. Biol. Rep. 5, 387-405

24. Keppler, L. D., Baker, C. J., and Atkinson, M. M. (1989) Phytopathology 79, 974-978

25. Slabas, A. R., Sidebottom, C. M., Hellyer, A., Kessell, R. M. J., and Tombs, M. P. (1986) Biochim. Biophys. Acta 877, 271-280

26. Gomez-Gomez, L., Felix, G., and Boller, T. (1999) Plant J. 18, 277-284

27. Eulgem, T., Rushton, P. J., Robatzek, S., and Somssich, I. E. (2000) Trends Plant Sci. 5, 199-206

28. Ulker, B., and Somssich, I. E. (2004) Curr. Opin. Plant Biol. 7, 491-498

29. Lamb, C., and Dixon, R. A. (1997) Annu. Rev. Plant. Physiol. Plant. Mol. Biol. 48, 251-275

30. Poghosyan, Z. P., Giannoulia, K., Katinakis, P., Murphy, D. J., and Hatzopoulos, P. (2005) Plant Physiol. Biochem. 43, 37-44

31. McMurry, L. M., Oethinger, M., and Levy, S. B. (1998) Nature 394, 531-532

32. Kater, M. M., Koningstein, G. M., Nijkamp, H. J., and Stuitje, A. R. (1994) Plant Mol. Biol. 25, 771-790

33. Mou, Z., He, Y., Dai, Y., Liu, X., and Li, J. (2000) Plant Cell 12, 405-418

34. Sasaki, Y., and Nagano, Y. (2004) Biosci. Biotechnol. Biochem. 68, $1175-1184$

35. Heath, R. J., Yu, Y. T., Shapiro, M. A., Olson, E., and Rock, C. O. (1998) J. Biol. Chem. 273, 30316-30320

36. Heath, R. J., White, S. W., and Rock, C. O. (2002) Appl. Microbiol. Biotechnol. 58, 695-703

37. Blakley, R. L. (1995) Adv. Enzymol. Relat. Areas Mol. Biol. 70, 23-102

38. Affleck, J. G., Neumann, K., Wong, L., and Walker, V. K. (2006) Toxicol. Sci. 89, 495-503

39. Felix, G., Duran, J. D., Volko, S., and Boller, T. (1999) Plant J. 18, 265-276

40. Gomez-Gomez, L., Bauer, Z., and Boller, T. (2001) Plant Cell 13, $1155-1163$

41. Shah, K., Russinova, E., Gadella, T. W., Jr., Willemse, J., and De Vries, S. C. (2002) Genes Dev. 16, 1707-1720

42. Testerink, C., and Munnik, T. (2005) Trends Plant Sci. 10, 368-375

43. van der Luit, A. H., Piatti, T., van Doorn, A., Musgrave, A., Felix, G., Boller, T., and Munnik, T. (2000) Plant Physiol. 123, 1507-1516

44. de Jong, C. F., Laxalt, A. M., Bargmann, B. O., de Wit, P. J., Joosten, M. H., and Munnik, T. (2004) Plant J. 39, 1-12

45. Lee, C. S., Kim, I. S., Park, J. B., Lee, M. N., Lee, H. Y., Suh, P. G., and Ryu, S. H. (2006) Nat. Cell Biol. 8, 477-484. 